\section{Canny Edge Detection Enhancement by Scale Multiplication}

\author{
Paul Bao, Lei Zhang, and Xiaolin Wu
}

\begin{abstract}
The technique of scale multiplication is analyzed in the framework of Canny edge detection. A scale multiplication function is defined as the product of the responses of the detection filter at two scales. Edge maps are constructed as the local maxima by thresholding the scale multiplication results. The detection and localization criteria of the scale multiplication are derived. At a small loss in the detection criterion, the localization criterion can be much improved by scale multiplication. The product of the two criteria for scale multiplication is greater than that for a single scale, which leads to better edge detection performance. Experimental results are presented.
\end{abstract}

Index Terms-Edge detection, scale multiplication, multiscale analysis.

\section{INTRODUCTION}

EDGES carry important information of an image. Numerous edge detection techniques have been proposed [1], [2], [3], [4], [5], [6], [7] [8], [9]. The common approach is to apply the first (or second) derivative to the smoothed image and then find the local maxima (or zero-crossings). Canny [2] first presented the well-known three criteria of edge detectors: good detection, good localization, and low spurious response and showed that the optimal detector for an isolated step edge should be the first derivative of Gaussian (FDOG). An error in Canny's localization criterion was fixed by Tagare and de Figueiredo [5] and by Koplowitz and Greco [6]. Recently, Demigny [4] analyzed in detail the optimal detection filters in discrete domain.

An important issue in edge detection is the scale of detection filter. Small-scaled filters are sensitive to edge signals but also prone to noise, whereas large-scaled filters are robust to noise but could filter out fine details. As suggested by Marr and Hildreth [1], multiple scales could be employed to describe and synthesize the varieties of edge structures. The idea of scale multiplication was first exploited by Rosenfeld in [3], where it is shown that the scale products can improve the edge localization. Mallat [10] illustrated mathematically that signals and noise have different singularities and edge structures present observable magnitudes along the scales, while noise decreases rapidly. With this observation, $\mathrm{Xu}$ et al. [11] proposed a wavelet-based spatially selective filtering technique by multiplying the adjacent scales. Sadler and Swami [12] applied the wavelet-multiscale-products to step detection and estimation and Bao and Zhang [13] presented a denoising scheme by thresholding the multiscale products.

Inspired by the work of Canny [2] and others [5], [6] on criteria of edge detection filters, we define the detection and localization criteria of the scale multiplication. We show that through scale multiplication the localization accuracy can be significantly improved with only a small loss in the detection criterion and the product of the two criteria for the scale multiplication is greater than that for a single scale, leading to superior edge detection results. A simple but efficient edge detector by scale multiplication is then proposed.

- P. Bao is with the Nanyang Technological University, Singapore, 639798. E-mail: aspbao@ntu.edu.sg.

- L. Zhang and X. Wu are with McMaster University, Hamilton, ON, Canada, L8G 4K1. E-mail: \{johnray,xwu\}@mail.ece.mcmaster.ca.

Manuscript received 26 May 2004; revised 14 Jan. 2005; accepted 14 Feb. 2005; published online 14 July 2005.

Recommended for acceptance by $R$. Chellappa.

For information on obtaining reprints of this article, please send e-mail to: tpami@computer.org, and reference IEEECS Log Number TPAMI-0262-0504.

\section{Criteria of Optimal Edge Detection Filters}

Suppose that an FIR filter $f(x)$ supported in $[-T, T]$ is used as the detector. The signal to be detected is a single step edge corrupted by noise: $W(x)=G(x)+n(x)$, where $n(x) \sim N\left(0, \sigma^{2}\right)$ is Gaussian white noise and $G(x)$ is a step edge whose magnitude is $A$ when $x \geq 0$. The response of $G(x)$ to $f(x)$ is $H_{G}(x)=\int_{-T}^{T} G(x-t) f(t) d t$ and that of $n(x)$ is $H_{n}(x)=\int_{-T}^{T} n(x-t) f(t) d t$ and then the response of $W(x)$ to $f(x)$ is $H_{W}(x)=H_{G}(x)+H_{n}(x)$. Considering the edges as the local maxima of $H_{W}(x)$, Canny [2] generalized three criteria to evaluate an edge detector:

1. Good detection. It is expected that at abscissa $x=0$, the signal-to-noise ratio (SNR) should be as high as possible. The criterion is:

$$
S N R=A\left|\int_{-T}^{0} f(x) d x\right| /\left(\sigma \sqrt{\int_{-T}^{T} f^{2}(x) d x}\right) .
$$

2. Good localization. The detected edge should be as close to location $x=0$ as possible. Denote by $y$ the local maximum position in $H_{W}(x)$. Canny approximated $y \approx H_{n}^{\prime}(y) / H_{G}^{\prime \prime}(0)$ and defined the criterion as the reciprocal of the standard deviation of $y$. He computed $E\left[H_{n}^{\prime 2}(y)\right]=\sigma^{2} \int_{-T}^{T} f^{\prime 2}(x) d x$. However, Tagare and Figueiredo [5] pointed out this expression is correct only if $H_{n}^{\prime}(x)$ is sampled at the same position for all realizations of $H_{n}(x)$. But, the location $y$ is not constant. In [6], Koplowitz and Greco gave another approximation of $y$ :

$$
y \approx-H_{n}^{\prime}(0) /\left(H_{G}^{\prime \prime}(0)+H_{n}^{\prime \prime}(0)\right) .
$$

Let $\varsigma=-H_{n}^{\prime}(0)$ and $\xi=H_{G}^{\prime \prime}(0)+H_{n}^{\prime \prime}(0)$. $\varsigma$ and $\xi$ are independent Gaussian variables. Denote by $\mu_{\varsigma}$ and $\mu_{\xi}$ and $\sigma_{\varsigma}^{2}$ and $\sigma_{\xi}^{2}$ the means and variances of them, respectively. The pdf of $y$ is [6]:

$$
\operatorname{Pr}(y)=\frac{1}{2 \pi \sigma_{\varsigma} \sigma_{\xi}} \cdot \frac{e^{-c}}{a}\left(1+b \sqrt{\pi / a} \cdot e^{b^{2} / a} \cdot \operatorname{erf}(b / \sqrt{a})\right),
$$

where $\operatorname{erf}(x)$ is the error function and $a=\frac{y^{2}}{2 \sigma_{\varsigma}^{2}}+\frac{1}{2 \sigma_{\varepsilon}^{2}}, b=-$ $\frac{\mu_{\varsigma} y}{2 \sigma_{\varsigma}^{2}}-\frac{\mu_{\xi}}{2 \sigma_{\epsilon}^{2}}$, and $c=\frac{\mu_{\varsigma}^{2}}{2 \sigma_{\varsigma}^{2}}+\frac{\mu_{\xi}^{2}}{2 \sigma_{\epsilon}^{2}}$. Note that $\int y^{2} \operatorname{Pr}(y) d y$ is infinite. Limiting the edge location between interval $[-T, T]$, we compute the variance of $y$ as $E\left[y^{2}\right]=\int_{-T}^{T} y^{2} \operatorname{Pr}(y) d y$. Thus, the good localization criterion is defined as

$$
L=1 / \sqrt{\int_{-T}^{T} y^{2} \operatorname{Pr}(y) d y} .
$$

3. Low spurious response. Since the input is a single step, the detector should not produce multiple maxima. Canny defined by

$$
x_{\max }(f)=2 \pi \sqrt{\int_{-T}^{T} f^{\prime 2}(x) d x} / \int_{-T}^{T} f^{\prime \prime} 2(x) d x
$$

the mean distance between two noise maxima and expected $x_{\max }(f)$ to be as big as possible.

In Canny's view, the optimal edge detection filter should maximize the product of detection and localization criteria with the constraint in low spurious response. However, Demigny [4] pointed out that the criterion of low spurious response is not essential and he further proved that the influence of this criterion is greatly reduced by thresholding operation and a good filter can be determined by only optimizing the detection-localization product. 


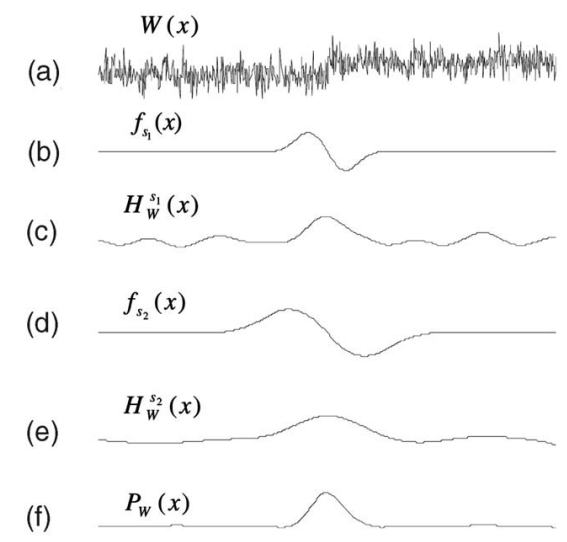

Fig. 1. (a) Noisy step edge $W(x)$. (b) The FDOG at scale $s_{1}$. (c) The response of $f_{s_{1}}(x)$ to $W(x)$. (d) The FDOG at scale $s_{2}$. (e) The response of $f_{s_{2}}(x)$ to $W(x)$. (f) The responses product of scales $s_{1}$ and $s_{2}$.

\section{ANALysis of Scale Multiplication IN EdGE DETECTION}

\subsection{Scale Multiplication}

Denote by $f_{s}(x)=f(x / s) / s$ the dilation of $f(x)$ by scale $s$. The support of $f_{s}(x)$ is $\left[-T_{s}, T_{s}\right]$, where $T_{s}=s T$. We denote the response of $W(x)$ to $f_{s}(x)$ as $H_{W}^{s}(x)=H_{G}^{s}(x)+H_{n}^{s}(x)$, where $H_{G}^{s}(x)$ and $H_{n}^{s}(x)$ are the responses of $G(x)$ and $n(x)$, respectively. In this paper, we take the FDOG $f(x)=-x \cdot e^{-x^{2} / 2}$ as the edge detection filter and then $f_{s}(x)=-x e^{-x^{2} /\left(2 s^{2}\right)} / s^{2}$. We use a small scale $s_{1}$ and a large scale $s_{2}$ to detect the step edge. The responses at the two scales are $H_{W}^{s_{1}}(x)$ and $H_{W}^{s_{2}}(x)$. The scale multiplication is defined as the product of $H_{W}^{s_{1}}(x)$ and $H_{W}^{s_{2}}(x)$

$$
P_{W}(x)=H_{W}^{s_{1}}(x) \cdot H_{W}^{s_{2}}(x)
$$

Fig. 1 shows a noisy step edge $W(x)$, the edge detection filters $f_{s_{1}}(x)$ and $f_{s_{2}}(x)\left(s_{2}=2 s_{1}\right)$, the responses at the two scales, and their products $P_{W}(x)$. We see that with scale $s_{1}$, the step edge is more accurately localized but some false local maxima are produced; with scale $s_{2}$, fewer false edges are detected but traded off with a decreased accuracy in edge location. $P_{W}(x)$ combines the advantages of the two scales. In $P_{W}(x)$, the step edge is much sharper compared with $H_{W}^{s_{2}}(x)$, while noise is better suppressed compared with $H_{W}^{s_{1}}(x)$. Intuitively, more robust detection results can be obtained if edges are determined as the local maxima in $P_{W}(x)$ after thresholding.

\subsection{The Detection Criterion}

The detection criterion proposed by Canny [2] is based on the linear property of convolution. In $P_{W}(x)$, however, nonlinearity is introduced by the multiplication operation. Similar to Canny's definition, the detection criterion is considered as the SNR of $P_{W}(x)$ at $x=0$, where the step edge occurs. If the system input is a noiseless signal $G(x), P_{W}(0)$ would be $H_{G}^{s_{1}}(0) \cdot H_{G}^{s_{2}}(0)$ and if the input is noise $n(x)$, the output would be $H_{n}^{s_{1}}(0) \cdot H_{n}^{s_{2}}(0)$. We define the detection criterion as

$$
S N R_{P}=\frac{\sqrt{\left|H_{G}^{s_{1}}(0) \cdot H_{G}^{s_{2}}(0)\right|}}{\sqrt{E\left[\left|H_{n}^{s_{1}}(0) \cdot H_{n}^{s_{2}}(0)\right|\right]}} .
$$

Note that, if $s_{1}=s_{2}$ (i.e., $P_{W}(x)$ is the square of $\left.H_{W}^{s_{1}}(x)\right), S N R_{P}$ is the same as the $S N R$ in (2.1) with $f(x)=f_{s_{1}}(x)$. Since $H_{G}^{s}(0)$ is invariant with scale $s$ and $H_{G}^{s}(0)=A$, we have $S N R_{P}=A$ / $\sqrt{E\left[\left|H_{n}^{s_{1}}(0) \cdot H_{n}^{s_{2}}(0)\right|\right]} \cdot H_{n}^{s_{1}}(0)$ and $H_{n}^{s_{2}}(0)$ are linear filtering outputs of Gaussian noise $n(x)$ and, thus, zero mean and jointly Gaussian distributed:

$$
\operatorname{Pr}\left(z_{1}, z_{2}\right)=\frac{1}{2 \pi \sigma_{1} \sigma_{2} \sqrt{1-\rho^{2}}} \exp \left\{-\frac{1}{2\left(1-\rho^{2}\right)}\left(\frac{z_{1}^{2}}{\sigma_{1}^{2}}-\frac{2 \rho z_{1} z_{2}}{\sigma_{1} \sigma_{2}}+\frac{z_{2}^{2}}{\sigma_{2}^{2}}\right)\right\},
$$

where

$$
\begin{aligned}
& \sigma_{1}=\sigma \cdot \sqrt{\int f_{s_{1}}^{2}(t) d t}=\sqrt[4]{\pi /\left(4 s_{1}^{2}\right)} \cdot \sigma \text { and } \\
& \sigma_{2}=\sigma \cdot \sqrt{\int f_{s_{2}}^{2}(t) d t}=\sqrt[4]{\pi /\left(4 s_{2}^{2}\right)} \cdot \sigma
\end{aligned}
$$

and $\rho$ is the correlation coefficient of $H_{n}^{s_{1}}(0)$ and $H_{n}^{s_{2}}(0)$ :

$$
\rho=\frac{\int f_{s_{1}}(t) f_{s_{2}}(t) d t}{\sqrt{\int f_{s_{1}}^{2}(t) d t} \cdot \sqrt{\int f_{s_{2}}^{2}(t) d t}}=\sqrt{\frac{2^{3} s_{1}^{3} s_{2}^{3}}{\left(s_{1}^{2}+s_{2}^{2}\right)^{3}}}
$$

Note that $\rho$ is invariant with the ratio $r=s_{2} / s_{1}$. The expectation of $\left|H_{n}^{s_{1}}(0) \cdot H_{n}^{s_{2}}(0)\right|$ is

$$
E\left[\left|H_{n}^{s_{1}}(0) \cdot H_{n}^{s_{2}}(0)\right|\right]=2 \sigma_{1} \sigma_{2}(\cos \alpha+\alpha \sin \alpha) / \pi,
$$

where $\alpha$ is given by $\sin \alpha=\rho,-\pi / 2<\alpha \leq \pi / 2$. Then, we can compute $S N R_{P}$ as

$$
S N R_{P}=\frac{A}{\sigma} \cdot \frac{\sqrt[4]{\pi s_{1} s_{2}}}{\sqrt{\cos \alpha+\alpha \sin \alpha}} .
$$

When ratio $r=s_{2} / s_{1}$ is fixed, $\rho$ is a constant and $\alpha$ is fixed. If $A / \sigma$ is also fixed, we see that $S N R_{P}$ is proportional to $\sqrt{s_{1}}$. This observation is similar tp that on the detection criterion $S N R$ in (2.1). Substituting $f_{s}(x)=-x e^{-x^{2} / s^{2}} / s^{2}$ for $f(x)$ in (2.1), we can compute that $S N R=\sqrt{s} \cdot(A / \sigma) \cdot \sqrt[4]{4 / \pi}$. Apparently, $S N R$ is also proportional to $\sqrt{s}$.

\subsection{The Localization Criterion}

Denote by $z$ the local maximum position of $P_{W}(x)$ near $x=0$. The derivative of $P_{W}(x)$ at $z$ is $P_{W}^{\prime}(z)=H_{W}^{s_{1}}(z) \cdot H_{W}^{s_{2}}(z)+H_{W}^{s_{1}}(z)$. $H_{W}^{\prime s_{2}}(z)=0$. By Taylor expansion of the derivatives of $H_{W}^{s}(z)$ and $H_{W}^{\prime s}(z)$ about $x=0$ and neglecting the $o\left(z^{2}\right)$ terms, we have

$$
z \approx \frac{-H_{n}^{\prime s_{1}}(0) H_{W}^{s_{2}}(0)-H_{n}^{\prime s_{2}}(0) H_{W}^{s_{1}}(0)}{H_{W}^{\prime \prime s_{1}}(0) H_{W}^{s_{2}}(0)+H_{W}^{\prime \prime} s_{2}(0) H_{W}^{s_{1}}(0)+2 H_{n}^{\prime s_{1}}(0) H_{n}^{\prime s_{2}}(0)} .
$$

We used $H_{G}^{\prime s}(0)=0$ in deriving the above equation. For expression convenience, we denote $\varsigma_{i}=H_{W}^{s_{i}}(0), \xi_{i}=H_{n}^{\prime s_{i}}(0)$, and $\eta_{i}=H_{W}^{\prime \prime s_{i}}(0)$, $i=1,2$ and then $z \approx \frac{-\xi_{1} \varsigma_{2}-\xi_{2} \varsigma_{1}}{\eta_{1} \varsigma_{2}+\eta_{2} \varsigma_{1}+2 \xi_{1} \xi_{2}}$.

All variables $\varsigma_{i}, \xi_{i}$, and $\eta_{i}$ are linear filtering outputs of noise $n(x)$, so they are jointly Gaussian. Let $\vec{X}=\operatorname{col}\left\{\varsigma_{1}, \varsigma_{2}, \xi_{1}, \xi_{2}, \eta_{1}, \eta_{2}\right\}$. The mean vector and covariance matrix of $\vec{X}$ are $\vec{M}=E[\vec{X}]$ and $\boldsymbol{\Omega}=E\left[(\vec{X}-\vec{M})(\vec{X}-\vec{M})^{T}\right]$. The pdf of $\vec{X}$ is [14]

$$
\left.\left.\operatorname{Pr}(\vec{X})=\frac{1}{(2 \pi)^{3} \sqrt{|\boldsymbol{\Omega}|}} \exp \left(-\frac{1}{2} \vec{X}-\vec{M}\right)^{T} \boldsymbol{\Omega}^{-1} \vec{X}-\vec{M}\right)\right),
$$

where $|\boldsymbol{\Omega}|$ is the determinant of matrix $\boldsymbol{\Omega}$. The elements of $\vec{M}=E[\vec{X}]$ are computed as $E\left[s_{i}\right]=H_{G}^{s_{i}}(0)=A, E\left[\xi_{i}\right]=0$, and $E\left[\eta_{i}\right]=H_{G}^{\prime \prime} s_{i}(0)=-A / s_{i}^{2}$. The elements of $\boldsymbol{\Omega}$ are

$$
\begin{aligned}
\operatorname{cov}\left(\varsigma_{i}, \varsigma_{j}\right) & =\sigma^{2} \int f_{s_{i}}(x) f_{s_{j}}(x) d x \\
& =\sigma^{2} \cdot \sqrt{2 \pi} s_{i} s_{j} /\left(s_{i}^{2}+s_{j}^{2}\right)^{1.5}, i, j=1,2
\end{aligned}
$$




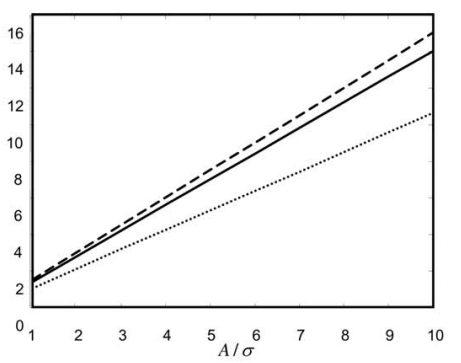

(a)

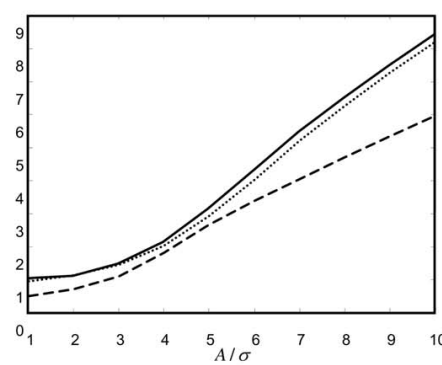

(b)

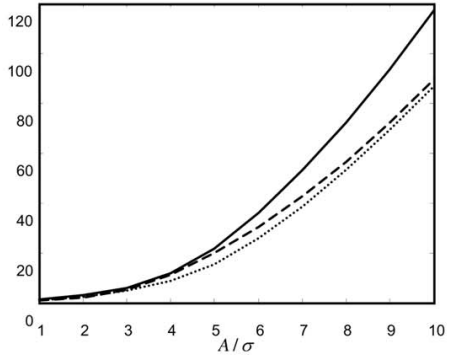

(c)

Fig. 2. When $s_{1}=2^{0}$ and $s_{2}=2^{1}$. (a) The curves $\Sigma_{1}$ (dotted), $\Sigma_{2}$ (dashed), and $\Sigma_{P}$ (solid). (b) The curves $\Lambda_{1}$ (dotted), $\Lambda_{2}$ (dashed), and $\Lambda_{P}$ (solid). (c) The curves $\Sigma_{1} \times \Lambda_{1}$ (dotted), $\Sigma_{2} \times \Lambda_{2}$ (dashed), and $\Sigma_{P} \times \Lambda_{P}$ (solid).

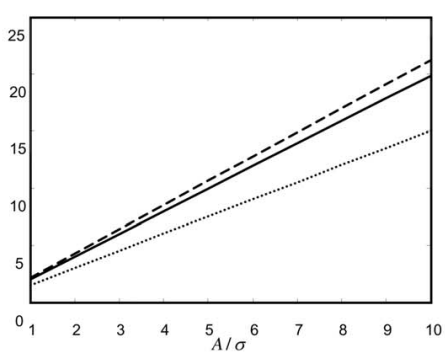

(a)

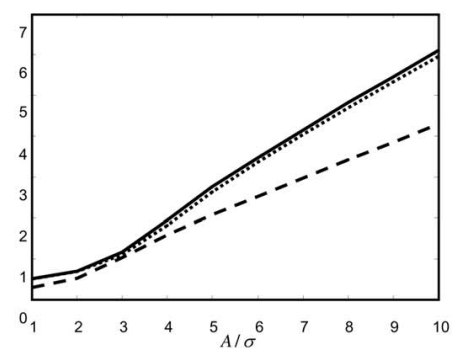

(b)

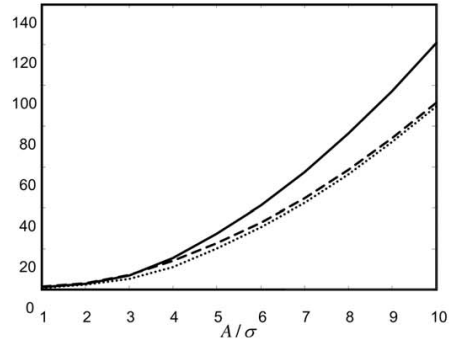

(c)

Fig. 3. When $s_{1}=2^{1}$ and $s_{2}=2^{2}$. (a) The curves $\Sigma_{1}$ (dotted), $\Sigma_{2}$ (dashed), and $\Sigma_{P}$ (solid). (b) The curves $\Lambda_{1}$ (dotted), $\Lambda_{2}$ (dashed), and $\Lambda_{P}$ (solid). (c) The curves $\Sigma_{1} \times \Lambda_{1}$ (dotted), $\Sigma_{2} \times \Lambda_{2}$ (dashed), and $\Sigma_{P} \times \Lambda_{P}$ (solid).

$\operatorname{cov}\left(\xi_{i}, \xi_{j}\right)=\sigma^{2} \int f_{s_{i}}^{\prime}(x) f_{s_{j}}^{\prime}(x) d x=\sigma^{2} \cdot 3 \sqrt{2 \pi} s_{i} s_{j} /\left(s_{i}^{2}+s_{j}^{2}\right)^{2.5}, i, j=1,2$,

$\operatorname{cov}\left(\eta_{i}, \eta_{j}\right)=\sigma^{2} \int f_{s_{i}}^{\prime \prime}(x) f_{s_{j}}^{\prime \prime}(x) d x=\sigma^{2} \cdot 15 \sqrt{2 \pi} s_{i} s_{j} /\left(s_{i}^{2}+s_{j}^{2}\right)^{3.5}, i, j=1,2$,

$\operatorname{cov}\left(\varsigma_{i}, \xi_{j}\right)=0, \operatorname{cov}\left(\varsigma_{i}, \eta_{j}\right)=0$, and $\operatorname{cov}\left(\eta_{i}, \xi_{j}\right)=0, i, j=1,2$.

In fact, we can write $\operatorname{Pr}(\vec{X})=\operatorname{Pr}\left(\varsigma_{1}, \varsigma_{2}\right) \cdot \operatorname{Pr}\left(\xi_{1}, \xi_{2}\right) \cdot \operatorname{Pr}\left(\eta_{1}, \eta_{2}\right)$ because $\varsigma_{i}, \xi_{i}$, and $\eta_{i}$ are mutually independent, where $\operatorname{Pr}(\bullet, \bullet)$ is the bivariate Gaussian distribution.

We limit the local maximum of $H_{W}^{s_{i}}(x)$ between $\left[-T_{s_{i}}, T_{s_{i}}\right]$. Suppose $s_{2}>s_{1}$, then $T_{s_{2}}>T_{s_{1}}$. Referring to Fig. 1, the edge is enhanced in $P_{W}(x)$ and we limit $y$, the local maximum of $P_{W}(x)$, between $\left[-T_{s_{1}}, T_{s_{1}}\right]$. The variance of $z$ can be computed by $E\left[z^{2}\right]=$ $\int_{-T_{s_{1}}}^{T_{s_{1}}} z^{2} \operatorname{Pr}(z) d z$ if we know $\operatorname{Pr}(z)$, the pdf of $z$. However, $z$ is a complex function of $\varsigma_{i}, \xi_{i}$, and $\eta_{i}$ and it is hard to obtain the analytic form of $\operatorname{Pr}(z)$. Instead, we compute numerically the variance of $z$ by using $\operatorname{Pr}(X)$ :

$$
E\left[z^{2}\right]=\iiint \iiint_{|z| \leq T_{s_{1}}} z^{2} \operatorname{Pr}(\vec{X}) d \varsigma_{1} d \varsigma_{2} d \xi_{1} d \xi_{2} d \eta_{1} d \eta_{2} .
$$

Finally, similar to (2.4), we define the localization criterion of $z$ as

$$
L_{P}=1 / \sqrt{E\left[z^{2}\right]} .
$$

\subsection{Numerical Comparison of $S N R_{P}, L_{P}$ with $S N R, L$}

$S N R_{P}$ is computed with (3.5) and $S N R$ defined in (2.1) is computed by $S N R=\sqrt{s} \cdot(A / \sigma) \cdot \sqrt[4]{4 / \pi}$. Localization criteria $L_{P}$ in (3.10) and $L$ in (2.4) are computed numerically. Since the Gaussian function decays rapidly, we set the support of $f(x)=$ $-x \cdot e^{-x^{2} / 2}$ to $[-4,4]$. Thus, the support of $f_{s}(x)=-x e^{-x^{2} / s^{2}} / s^{2}$ is
$[-4 s, 4 s]$, i.e., $T_{s}=4 s$. Parameters $\mu_{\varsigma}, \mu_{\xi}, \sigma_{\varsigma}$, and $\sigma_{\xi}$ in probability function $\operatorname{Pr}(y)$ (2.3) are computed as

$$
\begin{aligned}
& \mu_{\varsigma}=E\left[H_{n}^{\prime}(0)\right]=0, \\
& \sigma_{\varsigma}=\sqrt{E\left[H_{n}^{\prime 2}(0)\right]}=\sigma \sqrt{\int_{-T_{s}}^{T_{s}} f_{s}^{\prime 2}(x) d x}=\sigma \sqrt{3 \sqrt{\pi} /\left(4 s^{3}\right)}, \\
& \mu_{\xi}=E\left[H_{G}^{\prime \prime}(0)\right]=A f_{s}^{\prime}(0)=-A / s^{2}, \\
& \sigma_{\xi}=\sqrt{E\left[H_{n}^{\prime \prime 2}(0)\right]}=\sigma \sqrt{\int_{-T_{s}}^{T_{s}} f_{s}^{\prime \prime 2}(x) d x}=\sigma \sqrt{15 \sqrt{\pi} /\left(8 s^{5}\right)} .
\end{aligned}
$$

Denote by $\Sigma_{1}$ and $\Sigma_{2}$ the $S N R$ values of $f_{s_{1}}(x)$ and $f_{s_{2}}(x)$ and by $\Lambda_{1}$ and $\Lambda_{2}$ the $L$ values of them. The corresponding values of criteria $S N R_{P}$ and $L_{P}$ are denoted by $\Sigma_{P}, \Lambda_{P}$. In the sequel, we set $s_{2}=2 \cdot s_{1}$ and compute $\Sigma_{1}, \Sigma_{2}, \Lambda_{1}, \Lambda_{2}, \Sigma_{P}$, and $\Lambda_{P}$ versus $A / \sigma$.

Fig. 2a plots the curves of $\Sigma_{1}, \Sigma_{2}$, and $\Sigma_{P}$ versus $A / \sigma$ when $s_{1}=2^{0}$. Fig. $2 \mathrm{~b}$ plots the corresponding curves of $\Lambda_{1}, \Lambda_{2}$, and $\Lambda_{P}$, and Fig. $2 c$ plots the products $\Sigma_{1} \times \Lambda_{1}, \Sigma_{2} \times \Lambda_{2}$, and $\Sigma_{P} \times \Lambda_{P}$. Figs. $3 a, 3 b$, and $3 \mathrm{c}$ plot these curves for $s_{1}=2^{1}$. Similar observations are made on other values of $s_{1}$. We see that $\Sigma_{P}$ is much greater than $\Sigma_{1}$ and is only slightly less than $\Sigma_{2}$. At the same time, $\Lambda_{P}$ is not only much higher than $\Lambda_{2}$, but also slightly higher than $\Lambda_{1}$. Finally, $\Sigma_{P} \times \Lambda_{P}$ is much higher than $\Sigma_{1} \times \Lambda_{1}$ and $\Sigma_{2} \times \Lambda_{2}$, meaning that scale multiplication significantly improves edge detection results.

\subsection{Thresholding}

In first-derivative-based edge detection, the gradient image should be thresholded to eliminate false edges produced by noise. With a single threshold $t$, some false edges may appear if $t$ is too small and some true edges may be missed if $t$ is too large. In [2], Canny proposed a double thresholding algorithm. After nonmaxima suppression, a low threshold $t_{l}$ and a high threshold $t_{h} \approx 2 t_{l}$ are applied to obtain double thresholded edge maps, $I_{l}$ and $I_{h}$. The algorithm selects edges in $I_{l}$ that link to the edges in $I_{h}$. The double 


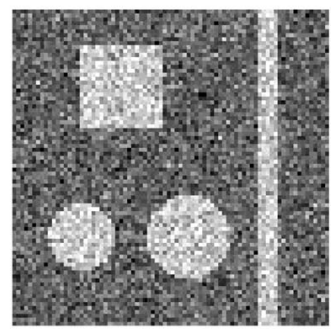

(a)

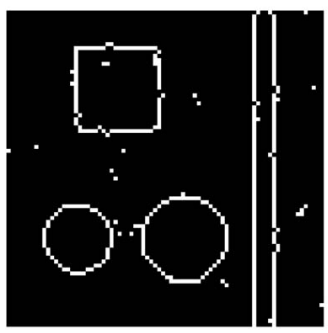

(b)

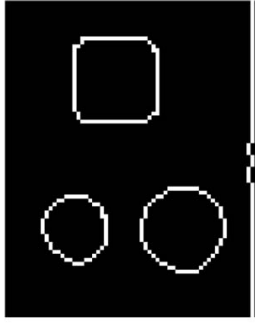

(c)

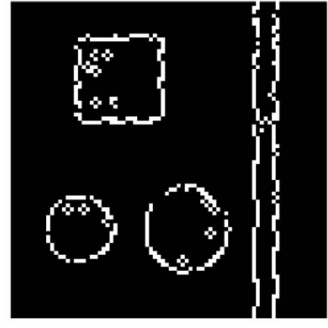

(d)

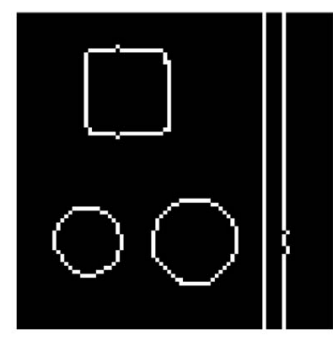

(e)

Fig. 4. (a) Noisy synthetic image. (b) Edge map by CED at a small scale $s_{1}$. (c) Edge map by CED at a large scale $s_{2}$. (d) Edge map by ADED. (e) Edge map by the proposed SMED with $s_{1}$ and $s_{2}$.

thresholding algorithm can also be applied to our scheme. Considering that edges and noise can be better distinguished in the scale products than in a single scale, however, we adopt the simple single thresholding strategy.

The edges are detected as the local maxima in $P_{W}(x)$. A significant edge at abscissa $x_{0}$ will occur at both the scales and the signs of $H_{W}^{s_{1}}\left(x_{0}\right)$ and $H_{W}^{s_{2}}\left(x_{0}\right)$ will be the same, so that $P_{W}\left(x_{0}\right)$ will be nonnegative. If $P_{W}(x)<0$, the point is considered as noise and filtered out. Suppose the input is pure noise $n(x)$ and then the products function is $P_{n}(x)=H_{n}^{s_{1}}(x) \cdot H_{n}^{s_{2}}(x)$. Variables $H_{n}^{s_{1}}(x)$ and $H_{n}^{s_{2}}(x)$ are jointly Gaussian with standard deviations $\sigma_{1}$ and $\sigma_{2}$, respectively, and correlation coefficient $\rho$. The pdf of their product, $P_{n}(x)$, is [14, p. 42]

$\operatorname{Pr}(z)=\frac{1}{\pi \Gamma(1 / 2) \sigma_{1} \sigma_{2} \sqrt{1-\rho^{2}}} \exp \left(\frac{\rho z}{\left(1-\rho^{2}\right) \sigma_{1} \sigma_{2}}\right) K_{0}\left(\frac{|z|}{\left(1-\rho^{2}\right) \sigma_{1} \sigma_{2}}\right)$,

where $\Gamma(t)=\int_{0}^{\infty} e^{-u} u^{t-1} d u$ is the Gamma function and $K_{0}$ is the modified Bessel function of the second kind with order zero. The standard deviation of $P_{n}(x)$ is

$$
\kappa=\sqrt{E\left[z^{2}\right]}=\sqrt{E\left[u^{2} v^{2}\right]}=\sqrt{1+2 \rho^{2}} \cdot \sigma_{1} \sigma_{2} .
$$

A threshold $t=c \cdot \kappa$ can suppress most of the $P_{n}(x)$ values by increasing $c$. In real applications, the input is the combination of noise and signal. The noise will be dominant in the filter response except for the significant edge structures. We find that setting $t_{P}=c \cdot \kappa, c>5$ leads to satisfactory results, i.e., suppressing most of the noise while well preserving the edges.

\subsection{Two Dimensions}

In 2D images, two scale product functions are needed. Denote by $f_{s}^{x}(x, y)$ and $f_{s}^{y}(x, y)$ the twodetection filters in $x$ and $y$ directions. Their responses to image $I$ are $H_{x}^{s}(x, y)$ and $H_{y}^{s}(x, y)$. The product functions are defined as $P_{I}^{x}(x, y)=H_{x}^{s_{1}}(x, y) \cdot H_{x}^{s_{2}}(x, y)$ and $P_{I}^{y}(x, y)=H_{y}^{s_{1}}$ $(x, y) \cdot H_{y}^{s_{2}}(x, y)$. For an edge point $\left(x_{0}, y_{0}\right), H_{x}^{s}\left(x_{0}, y_{0}\right)$, or $H_{y}^{s}\left(x_{0}, y_{0}\right)$ will have the same sign at adjacent scales $s_{1}$ and $s_{2}$ so both $P_{I}^{x}\left(x_{0}, y_{0}\right)$ and $P_{I}^{y}\left(x_{0}, y_{0}\right)$ will be nonnegative and the orientation information of the edge is lost, which should be recovered from $H_{x}^{s_{1}}\left(x_{0}, y_{0}\right)$ and $H_{y}^{s_{1}}\left(x_{0}, y_{0}\right)$. Setting the points with $P_{I}^{x}(x, y)<0$ (or $\left.P_{I}^{y}(x, y)<0\right)$ to 0 , the modulus and angle of point $(x, y)$ are defined as

$$
\begin{aligned}
M_{I}(x, y) & =\sqrt{P_{I}^{x}(x, y)+P_{I}^{y}(x, y)} \text { and } \\
\left.A_{(} x, y\right) & =\arctan \left(\frac{\operatorname{sgn}\left(H_{y}^{s_{1}}(x, y)\right) \cdot \sqrt{P_{I}^{y}(x, y)}}{\operatorname{sgn}\left(H_{x}^{s_{1}}(x, y)\right) \cdot \sqrt{P_{I}^{x}(x, y)}}\right) .
\end{aligned}
$$

As in the Canny edge detector, an edge point is asserted wherever $M_{I}(x, y)$ has a local maximum in the direction given by $A_{I}(x, y)$. The modulus map $M_{I}(x, y)$ should be thresholded to remove noise. Referring to Section 3.5, the threshold applied to $P_{I}^{x}(x, y)$ is $t_{P}^{x}=c \cdot \kappa_{x}, c>5$. The threshold $t_{P}^{y}$ applied to $P_{I}^{y}(x, y)$ is obtained similarly. We set the threshold to $M_{I}(x, y)$ as $t_{P}=\sqrt{t_{P}^{x}+t_{P}^{y}}$.

\section{EXPERIMENTAL RESULTS}

We experimented the proposed technique on a synthetic image and two natural images. The synthetic image, in which the true edges are known, is used to test the edge detection performance by some quantitative measurement. With the scale multiplication strategy, we choose the larger scale big enough to keep the false edge rate low, while achieving high edge location accuracy by multiplying a small scale. Let $s_{2}=r \cdot s_{1}$ with $r>1$. Too small an $r$ would not incorporate sufficient information of two different scales, whereas too large an $r$ would reduce the correlation of the two scales. $r=2$ is a good choice and it is convenient to discrete implementation. Suppose at scale $s_{1}$ the discrete filter length is $l_{1}$, then at scale $s_{2}=2 s_{1}$ the filter length is $l_{2}=2 l_{1}+2$. At the finest scale, $s=2^{0}$, we discretize the FDOG filter to be $f_{1}=[-1,1]$, and at the second scale $s=2^{1}$, the filter is $f_{2}=[-1,-3,-2,2,3,1] / 4$, etc. The proposed edge detector (we denote it as SMED) is compared with the Canny edge detector (CED) and the recently proposed anisotropic diffusion edge detector (ADED) by Black et al. [8]. The source code of method ADED is obtained from the Web site ftp://figment.csee.usf.edu/pub/Edge_Comparison/ source_code/anisotropic.tar.gz. In ADED, there is one parameter, threshold $t$. We set scale $s_{1}=2^{2}$ and scale $s_{2}=2^{3}$ in implementing the SMED and CED in the following experiments. In CED, we fix the low threshold to be half of the high one and set the high threshold as $t_{j}=\lambda \sigma_{j}$ at scale $s_{j}$, where $\sigma_{j}$ (referring to (3.2)) is the noise level at that scale and constant $\lambda$ is used to control the threshold. In the following experimental results of CED and ADED, we adjusted the threshold until a visually best edge map is obtained. 
TABLE 1

The Figure of Merit Values of the Edge Maps in Fig. 4

\begin{tabular}{|c|c|c|c|c|}
\hline Edge Maps & Fig. 4 (b) & Fig. 4 (c) & Fig. 4 (d) & Fig. 4 (e) \\
\hline$F$ & 0.8908 & 0.8413 & 0.8022 & 0.9756 \\
\hline
\end{tabular}

Fig. 4a shows a noisy synthetic image with one square, two circles, and two neighboring step edges in it. The added noise level is $\sigma=35$. Figs. $4 \mathrm{~b}$ and $4 \mathrm{c}$ illustrate the edge maps generated by CED at the two scales. The constant $\lambda$ is set to 2.4 and 2.0 , respectively, at the two scales. We see that, at the small scale there are many false edges caused by noise, while at the large scale the edge localization accuracy is decreased as a tradeoff of low false-edge-rate. Note that the distance between the two neighboring step edges in Fig. 4c is mistakenly amplified due to the interference of adjacent edge structures when detector scale is increased. Fig. $4 \mathrm{~d}$ shows the ADED results. The threshold is $t=1$. It is seen that ADED does not suppress noise well. Fig. 4e is the edge map detected by SMED with $c=6$. We see that the results remove most of the false edges and achieve very high edge localization performance. The shapes of the objects are precisely detected.

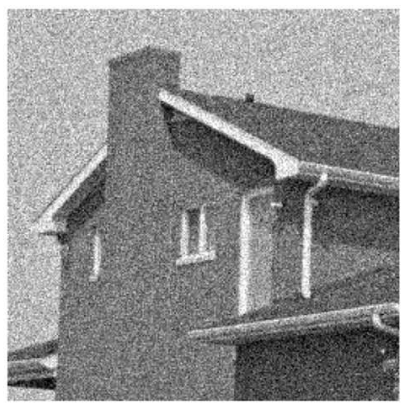

(a)

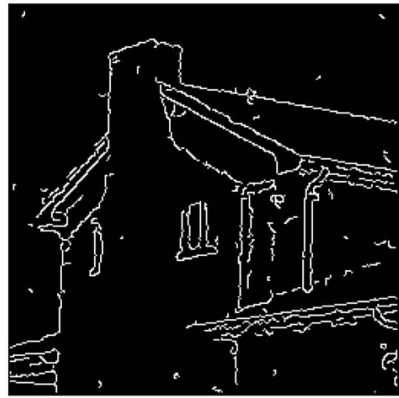

(b)

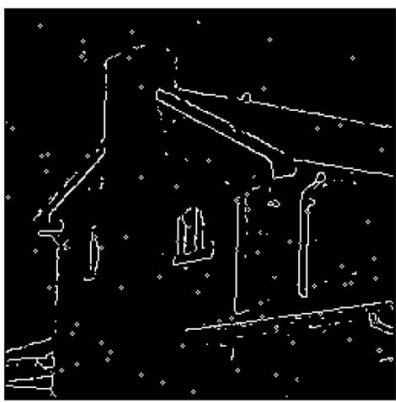

(d)

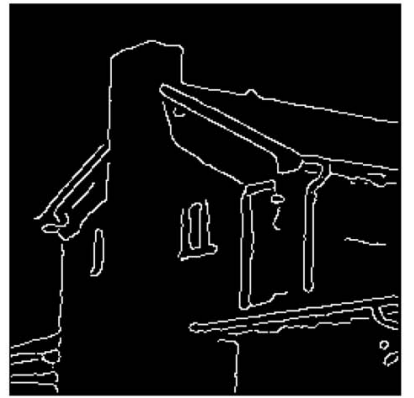

(c)

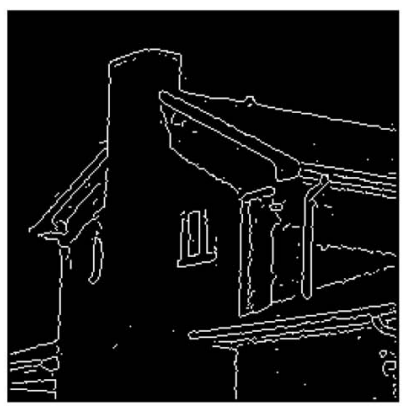

(e)
Fig. 5. (a) Noisy image House. (b) Edge map by CED at a small scale $s_{1}$. (c) Edge map by CED at a large scale $s_{2}$. (d) Edge map by ADED. (e) Edge map by the proposed SMED with $s_{1}$ and $s_{2}$.

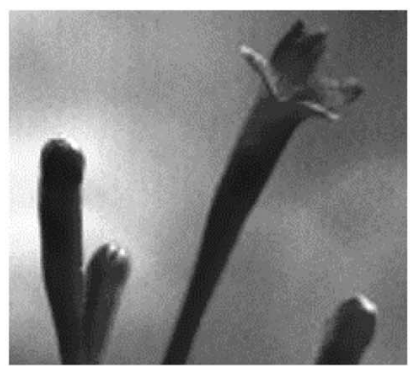

(a)

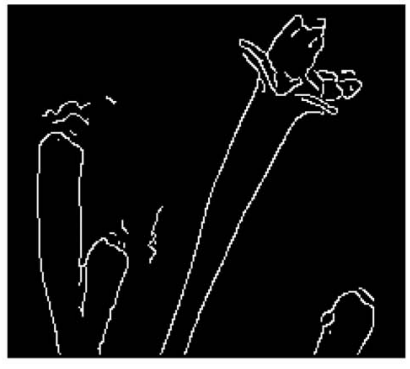

(b)

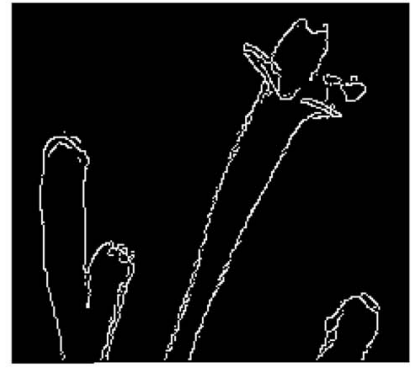

(d)

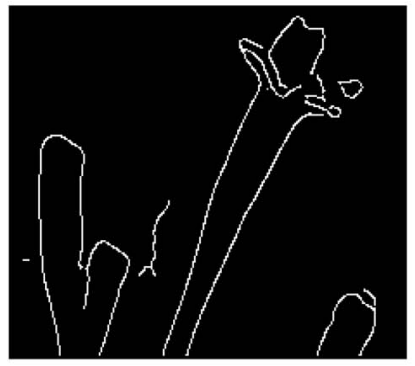

(c)

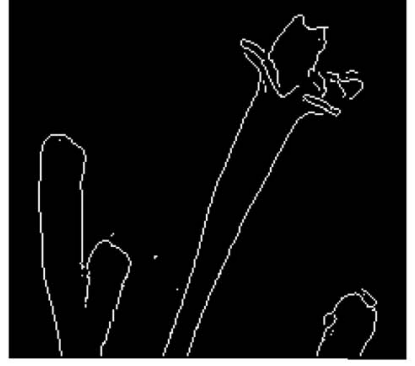

(e)
Fig. 6. (a) Image Flower. (b) Edge map by CED at a small scale $s_{1}$. (c) Edge map by CED at a large scale $s_{2}$. (d) Edge map by ADED. (e) Edge map by the proposed SMED with $s_{1}$ and $s_{2}$.

To objectively compare the edge detection results for this synthetic image, the measurement figure of merit $F$ proposed by Pratt [15] is used to evaluate the performance quantitatively:

$$
F=\frac{1}{\max \left\{N_{I}, N_{A}\right\}} \sum_{k=1}^{N_{A}} \frac{1}{1+\alpha d^{2}(k)},
$$

where $N_{I}$ is the number of the actual edges and $N_{A}$ is the number of the detected edges. $d(k)$ denotes the distance from the $k$ th actual edge to the corresponding detected edge. $\alpha$ is a scaling constant set to $1 / 9$ as in Pratt's work. The greater the $F$, the better the detection results. In Table 1 , the values of $F$ for the edge maps in Figs. $4 b, 4 c$, $4 \mathrm{~d}$, and $4 \mathrm{e}$ are listed.

In Fig. 5, the experimental results on a $256 \times 256$ noisy House image (added noise level is $\sigma=25$ ) by the three schemes are shown. The constant $\lambda$ in CED is 2.6 and 2.0, respectively, at the two scales. The threshold in ADED is $t=0.8$. The threshold constant in SMED is $c=6$. The last experiment is on a naturally noisy image. The image Flower taken by a digital camera is corrupted by background noise in the acquisition process. The noise level is estimated as $\sigma=11$. Fig. 6 showed the edge maps. The constant $\lambda$ in CED is 2.5 and 2.1, respectively. The threshold in ADED is $t=1.8$. The constant in SMED is set to $c=7$. It can be seen that the proposed scheme achieved very good results with few false edges and high localization accuracies. 


\section{Conclusion}

We developed a scale multiplication-based scheme to improve the performance of traditional Canny edge detector. Taking the advantage of similarities in the filter's responses at adjacent scales, the new scheme multiplies the responses to enhance edge structures while diluting noise and detect the edges as the local maxima in the scale products. Our theoretical analyses show that scale multiplication can improve the edge localization accuracy and then yield better edge detection results. Experiments on synthetic and natural images were made to test the proposed method.

\section{REFERENCES}

[1] D. Marr and E. Hildreth, "Theory of Edge Detection," Proc. Royal Soc. London, vol. 207, pp. 187-217, 1980.

[2] J. Canny, "A Computational Approach to Edge Detection," IEEE Trans. Pattern Analysis and Machine Intelligence, vol. 8, pp. 679-698, 1986.

[3] A. Rosenfeld, "A Nonlinear Edge Detection Technique," Proc. IEEE, pp. 814816, May 1970.

[4] D. Demigny, "On Optimal Linear Filtering for Edge Detection," IEEE Trans. Image Processing, vol. 11, pp. 728-1220, July 2002.

[5] H.D. Tagare and R.J.P. de Figueiredo, "On the Localization Performance Measure and Optimal Edge Detection," IEEE Trans. Pattern Analysis and Machine Intelligence, vol. 12, no. 12, pp. 1186-1190, Dec. 1990.

[6] J. Koplowitz and V. Greco, "On the Edge Location Error for Local Maximum and Zero-Crossing Edge Detectors," IEEE Trans. Pattern Analysis and Machine Intelligence, vol. 16, no. 12, pp. 1207-1212, Dec. 1994.

[7] M.C. Shin, D.B. Goldgof, K.W. Bowyer, and S. Nikiforou, "Comparison of Edge Detection Algorithms Using a Structure from Motion Task," IEEE Trans. System, Man, and Cybernetics-Part B: Cybernetics, vol. 31, pp. 589-601, Aug. 2001.

[8] M. Black, G. Sapiro, D. Marimont, and D. Heeger, "Robust Anisotropic Diffusion," IEEE Trans. Image Processing, vol. 7, pp. 421-432, Mar. 1998.

[9] H. Jeong and C.I. Kim, "Adaptive Determination of Filter Scales for Edge Detection," IEEE Trans. Pattern Analysis and Machine Intelligence, vol. 14, no. 5, pp. 579-585, May 1992.

[10] S. Mallat, A Wavelet Tour of Signal Processing. Academic Press, 1998.

[11] Y. Xu et al., "Wavelet Transform Domain Filters: A Spatially Selective Noise Filtration Technique," IEEE Trans. Image Processing, vol. 3, pp. 747-758, Nov. 1994.

[12] B.M. Sadler and A. Swami, "Analysis of Multiscale Products for Step Detection and Estimation," IEEE Trans. Information Theory, vol. 45, pp. 10431051, Apr. 1999.

[13] P. Bao and L. Zhang, "Noise Reduction for Magnetic Resonance Image via Adaptive Multiscale Products Thresholding," IEEE Trans. Medical Imaging, vol. 22, pp. 1089-1099, Sept. 2003

[14] K.S. Miller, Multidimensional Gaussian Distributions. New York: Wiley, 1964.

[15] W.K. Pratt, Digital Image Processing, second ed. John Wiley \& Sons, Apr. 1991.

$\triangleright$ For more information on this or any other computing topic, please visit our Digital Library at www.computer.org/publications/dlib. 